\title{
InVestigating The Performance of Various CHANNEL ESTIMATION TECHNIQUES FOR MIMO-OFDM SYSTEMS USING MATLAB
}

\author{
Woud M. Abed and Raghad K. Mohammed \\ Department of Basic Science, College of Dentistry \\ University of Baghdad, Baghdad, Iraq
}

\begin{abstract}
This paper simulates and investigates the performance of four widely-used channel estimation techniques for MIMO-OFDM wireless communication systems; namely, super imposed pilot (SIP), comb-type, spacetime block coding (STBC), and space-frequency block coding (SFBC) techniques. The performance is evaluated through a number of MATLab simulations, where the bit-error rate (BER) and the mean square error (MSE) are estimated and compared for different levels of signal-to-noise ratio (SNR). The simulation results demonstrate that the comb-type channel estimation and the SIP techniques overwhelmed the performance of the STFC and STBC techniques in terms of both bit-error rate (BER) and mean square error (MSE).
\end{abstract}

\section{KEYWORDS}

MIMO-OFDM, pilot-based channel estimation, pilot allocation, SIP, comb-type, STBC, SFBC, MATLab.

\section{INTRODUCTION}

One of the most challenging topics in today wireless communication systems is how to accomplish high data transmission rate and maintain satisfactory quality-of-service (QoS) that can meet the exponentially growing users and applications demands. This is a challenging topic because of the limited spectrum and the channel fading caused by multipath components in the wireless channel. One of the promising solutions to this problem is to combine the concepts of multiple-input multiple-output (MIMO) with orthogonal frequency division multiplexing (OFDM); which led to the emergent of the new MIMO-OFDM wireless communication systems $[1,2]$.

The MIMO technology implies the use of multiple antennas at the transmitter and the receiver sides of the wireless link, which significantly increases the data transmission rates [3]. OFDM implies a multicarrier transmission and frequency division multiplexing, where a single data stream is transmitted over several low rate subcarriers, placed orthogonal to each other to provide high data transmission rate with minimum error, and enough robustness to radio channel impairments. Practically, the transmitted signals of MIMO-OFDM systems benefit from the multi-path delay tolerance and immunity to frequency selective channel fading of MIMO, as well as the high data transmission rate of OFDM $[4,5]$.

Channel estimation is one of the main requirements of MIMO-OFDM systems. Therefore, a number of channels estimation techniques for MIMO-OFDM systems have been developed [68].In order to be able to select between these techniques, it is necessary to investigate their performance in different working environments. Computer simulation is well-recognized as the most feasible solution as practical investigations are complicated, costly, and time consuming $[9,10]$. 
This paper simulates and investigates the performance of four different channel estimation techniques for MIMO-OFDM systems; these are: super imposed pilot (SIP) [11], comb-type [12], space-time block coding (STBC) [13], and space-frequency block coding (SFBC) [14] techniques. The performance is evaluated through a number of MATLab simulations, where the bit-error rate (BER) and the mean square error (MSE) are estimated and compared for different levels of signal-to-noise ratio (SNR) [5]. The simulation results demonstrate that the comb-type and SIP provides better performance in terms of both BER and MSE over the STBC and SFBC especially with SNR.

The paper is divided into 6 sections. This section presents the main theme of the paper. A review of some of the most recent and related works on simulation and investigation of the performance of MIMO-OFDM systems, using MATLab, are given in Section 2.Section 3 explains the basic principles of MIMO-OFDM and the main concepts of the pilot-based channel estimation techniques studied in this paper. The simulated MIMO-OFDM system model is described in Section 4. The results and discussions are given in Section 5. Finally, in Section6 some conclusions are presented and a number of recommendations for future are pointed-out.

\section{LITERATURE REVIEW}

This section presents a review of some of the most recent development and performance evaluation of MIMO-OFDM systems using MATLab [1,9].

Chourasiya and Saraf [6] investigated the effect of different modulation techniques (e.g., BPSK, QPSK, 16 PSK and 16 QAM)on the BER performance of a 2x2 MIMO-OFDM system using MATLab. Their results conclude that for the same SNR, the BER of BPSK is lower than QPSK, 16 PSK and 16 QAM.Similarly, using MATLab,Sharma and Kaur [7] and Jangalwa [15] showed that MIMO-OFDM can provide better BER performance than OFDM systems. Katgi [8] investigated the effect of the multi-antenna combination (e.g., $2 \times 1,2 \times 2,4 \times 1$ and $4 \times 2$ ), and modulation technique (e.g., BPSK and QPSK) on the performance of MIMO-OFDM systems.

Rashid and Hossain [16] used MATLab to investigate the performance of MIMO-OFDM systemsin5G networks, with the aim of achieving the lowest and optimum BER while increasing the system capacity. Shrivastava and Trivedi [14] implemented and analyzed the performance of MIMO-OFDM system using space-time-frequency (STF) coding and random beam forming using MATLab. They found that combining beam forming with STF coding provides lower BER over STF alone, as well as the combined STBC-beam forming.

Achra et. al. [3] discussed and analyzed the BER performance of MIMO-OFDM system with two different equalizers (ZF and MMSE) for various modulation techniques (e.g., BPSK, QPSK, 16QAM, and 64-QAM) using multipath fading channels, namely, additive white Gaussian noise (AWGN) and Rayleigh and Rician channels. The simulation results show that MMSE equalizer achieves a lower BER than ZF for various SNR. Similar study by Pandey et. al. [17] analyzed the performance of a QPSK modulation MIMO-OFDM system with AWGN, Rayleigh, and Rician channels. They demonstrated that MIMO-OFDM with STBC using $2 \times 2$ antenna configurations and 512 FFT length has better performance in terms of BER than the other systems.

Sethy and Swain [18] found that for different fading channel, MIMO achieves full diversity using OSTBC encoder to overcome fading effect of channel, and OFDM reduces inter symbol interference (ISI) for higher data rate and higher spectral efficiency. Based on the simulation results, the BER performance of MIMO system is better than that of MIMO-OFDM system, but MIMO-OFDM system is spectrally more efficient. 
Reddy and Lakshmi [19] explored how MIMO increases channel capacity using a spatial modulation technique that combines the high multiplexing gain provided by the spatial modulation and transmit-diversity gain provided by the STBC technology. They explored two different STBC schemes (Alamouti's STBC and orthogonal STBC). The two schemes demonstrate higher data rate or longer transmit range without requiring additional bandwidth or transmit power.

Pathak and Sharma [20] investigated and compared the performance of two major types of pilot arrangement such as block-type and comb-type pilot, using the least square error (LSE) and minimum mean square error (MMSE) channel estimators. Patil and Jadhav [21] compared the performance of channel estimation algorithm and highlighted the channel estimation technique based on pilot-aided block-type training symbols using LS and MMSE algorithm.

Jain and Nandal [22] analyzed and compared the performance of channel estimation of MIMO communication systems using STBC, SFBC, and STFBC techniques under various fading channels. The performance is evaluated through a number simulations using MATLab. Daoud et. al [23] developed a new algorithm to enhance the performance of the speaker verification over the communication systems based MIMO-OFDM techniques.

In conclusion, the major advantages of MIMO-OFDM systems are: (i) substantial increase in channel capacity, which immediately translates to higher data throughput, (ii) significant improvement in data transmission reliability, i.e., very low bit error rate (BER). These advantages can be achieved without any expansion in the required bandwidth or increase in the transmit power.

\section{Pilot-Based Channel Estimation TechniQues}

High spectral efficiency and improved link reliability are two of the major challenges in wireless communications systems. The wireless channel constitutes a hostile propagation medium, which suffers from the addition of destructive multipath components (fading) and interference from other users. Diversity provides the receiver with several replicas of the transmitted signal and is therefore a powerful approach to alleviate fading and interference and thereby improve link reliability and channel capacity [1].

The use of spatial (or antenna) diversity has become very popular in recent years, which is mostly due to the fact that it can be easily implemented introducing any loss in spectral efficiency. Antenna diversity can be implemented at the receiving and transmitting sides. The use of multiple antennas at both ends of a wireless link (MIMO) has a great potential of achieving extraordinary data rates. On the other hand, OFDM can significantly reduce receiver complexity in wireless systems. Thus, combining MIMO with OFDM technologies to develop what is referred to as MIMO-OFDM system seems to be an attractive solution for future wireless systems [2].

Channel estimation in MIMO-OFDM systems is more complicated in comparison to that in single-input single-output OFDM (SISO-OFDM) systems [25]. Therefore, the techniques that are used in SISO-OFDM cannot be simply extended and used in MIMO-OFDM, because the superposition of signals from multiple transmits antennas makes decoupling pilot signals more complicated.

There are a number of pilot-based channel estimation techniques that have been developed for MIMO-OFDM systems over quasi-static channel $[6,18,19]$. This section discusses four of them, namely: SIP, comb-type, STBC, and SFBC channel estimation techniques. 


\subsection{SIP Channel Estimation Technique}

SIP channel estimation is not a straightforward approach compared to the following three schemes, which is inflexible in the pilot allocation and difficult to extend to the arbitrary number of transmit antennas. It has been recognized that SIP can save the subcarriers occupied by the pilots as depicted in Figure 1. In other words, this method estimates the MIMO-OFDM channel without employing additional pilots in the frequency domain, which improves the spectral efficiency. Thus, the main advantages of this SIP based channel estimation are: high spectral efficiency, low complexity, and no prior knowledge about channels and noise required. Description of the SIP based channel estimation technique can be found in $[11,26]$.

\subsection{Comb-Type Channel Estimation Technique}

The comb-type channel estimation for MIMO-OFDM systems is similar to these techniques employed in a single antenna scenario, due to the comb structure in the frequency and space domain. The diagram of such pilot allocation is shown in Figure2, which eliminates the effects of inter-antenna interference using additional null subcarriers. Hence, the SISO techniques can be extended to MIMO-OFDM systems, because the pilot pattern for each pair of transmit and receive antenna is similar to that of SISO-OFDM systems $[12,25]$.

Furthermore, the positions of pilots can be readily placed in the frequency and space domain as compared to SIP allocation. However, the comb-type channel estimation over very frequency selective channels requires more pilots in the sense that the more null subcarrier are employed, which reduces the spectral efficiency.

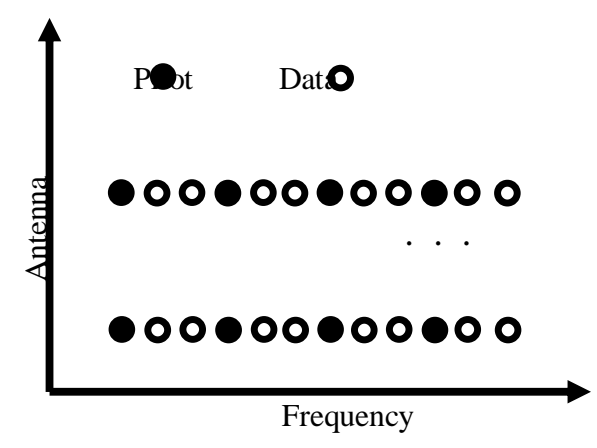

Figure 1. The diagram of SIP.

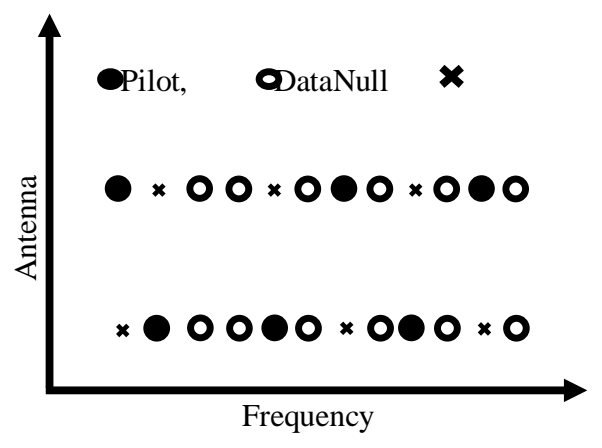

Figure 2. The diagram of comb-type.

\subsection{STBC Channel Estimation Technique}

STBC is a technique used in wireless communication to transmit multiple copies of a data stream across a number of antennas, to exploit the various received versions of data to improve the reliability of data transfer. STBC has emerged as one of the major techniques to exploit the MIMO benefit. Both spatial and temporal diversities are achieved in STBC, also it offers simple decoding with the use of maximum likelihood detection algorithm at the receiver [13].

STBC can be used in the channel estimation for MIMO-OFDM systems with the assumption that the channels remain constant in the time or frequency domain during several OFDM symbols. The assumption may not be practical in some scenarios, and the performance will be significantly affected if the channels become more selective [14]. 


\subsection{SFBC Channel Estimation Technique}

SFBC is the same as STBC is a technique used in wireless communications to transmit multiple copies of a data stream across a number of antennas and to exploit the various received versions of the data to improve the reliability of data transfer. SFBC involves coding across space and frequency, which is often referred to as space-frequency coding (SFC) [14]. A way to do SFC is to take the space-time codes (STC) (e.g., Alamouti code), and apply them in the frequency dimension instead of time dimension. That is, instead of mounting the space-time coded symbols on multiple time slots, they are mounted on multiple OFDM subcarriers. SFBC is similar to STBC it can be used in the channel estimation for MIMO-OFDM systems with the assumption that the channels remain constant in the time or frequency domain during several OFDM symbols.

\section{MIMO-OFDM SYSTEM MODEL}

The system model that is simulated and investigated is described as follows: An un-coded spatial multiplexing MIMO-OFDM system with $N_{s}$ subcarriers, $N_{t}$ transmit and $N_{r}$ receive antennas, where $N_{t} \leq N_{r}$, as shown in Figure3. In Figure (3, the modulated symbols from the modulators (MOD) and pilots are inserted into data subcarriers and pilot subcarriers via pilot allocation. The output of pilot allocation is then passed through inverse fast Fourier transform (IFFT) and appended with cyclic prefix (CP) for transmission. At the receiver, the $\mathrm{CP}$ is removed and the truncated received signals without $\mathrm{CP}$ are then passed through fast Fourier transform (FFT). The pilots are used for channel estimation, and the detection is performed with the estimated channels. The transmitted symbols are recovered through the demodulators (DEMOD) with the output of detection.

\section{RESULTS AND DISCUSSIONS}

In order to compare the performance of the channel estimation techniques described in Section 3, a number of simulations have been performed using MATLab. In these simulations, we consider a $2 \times 2$ MIMO-OFDM system, number of subcarrier $\left(N_{s}\right)$ is 128 , number of pilots uniformly distributed $\left(N_{p}\right)$ is 16 , channel length $(L)$ is 8 , normalized Doppler frequency $\left(f_{d} T_{\text {OFDM }}\right)$ is $10^{-2}$, and channel generation model is Jake's model. For comb-type channel estimation, 8 pilots and 8 null subcarriers are employed for fair comparison.

The performance is evaluated in terms of two widely-used performance measures; these are: the bit-error rate (BER) and the square mean error (MSE). BER is the number of bits that are erroneously received or altered (due to noise, interference, distortion or bit synchronization) divided by the total number of bits transmitted over the channel for a certain period of time. MSE is the average of the square of the error. The BER and MSE are investigated against an interesting input parameter, namely, the signal-to-noise ratio (SNR), which is defined as the ratio of signal power to the noise power. Often it is expressed in decibels and calculated by dividing the value of the main signal by the value of the noise, take the common logarithm of the result, and then multiply the result by 20 [5].

The BER and MSE performance are given in Figs. (4) and (5), respectively. In general, it can be seen from Figure 4 that the BER performance decreases with increasing SNR for all techniques. All techniques provides almost the same BER up to $\mathrm{SNR}=5 \mathrm{~dB}$. For $\mathrm{SNR}>5 \mathrm{~dB}$, SIP and combtype maintain almost the same BER, where both performs better than STBC and SFBC as they accomplish less BER. While the STBC provides the worst performance as it accomplishes the highest BER, and the BER performance is worsen with increasing SNR. The SFBC almost 
provides a moderate BER performance between the SIP and comb-type and the STBC. The BER performance of comb-type channel estimation is slightly better than that of SIP.

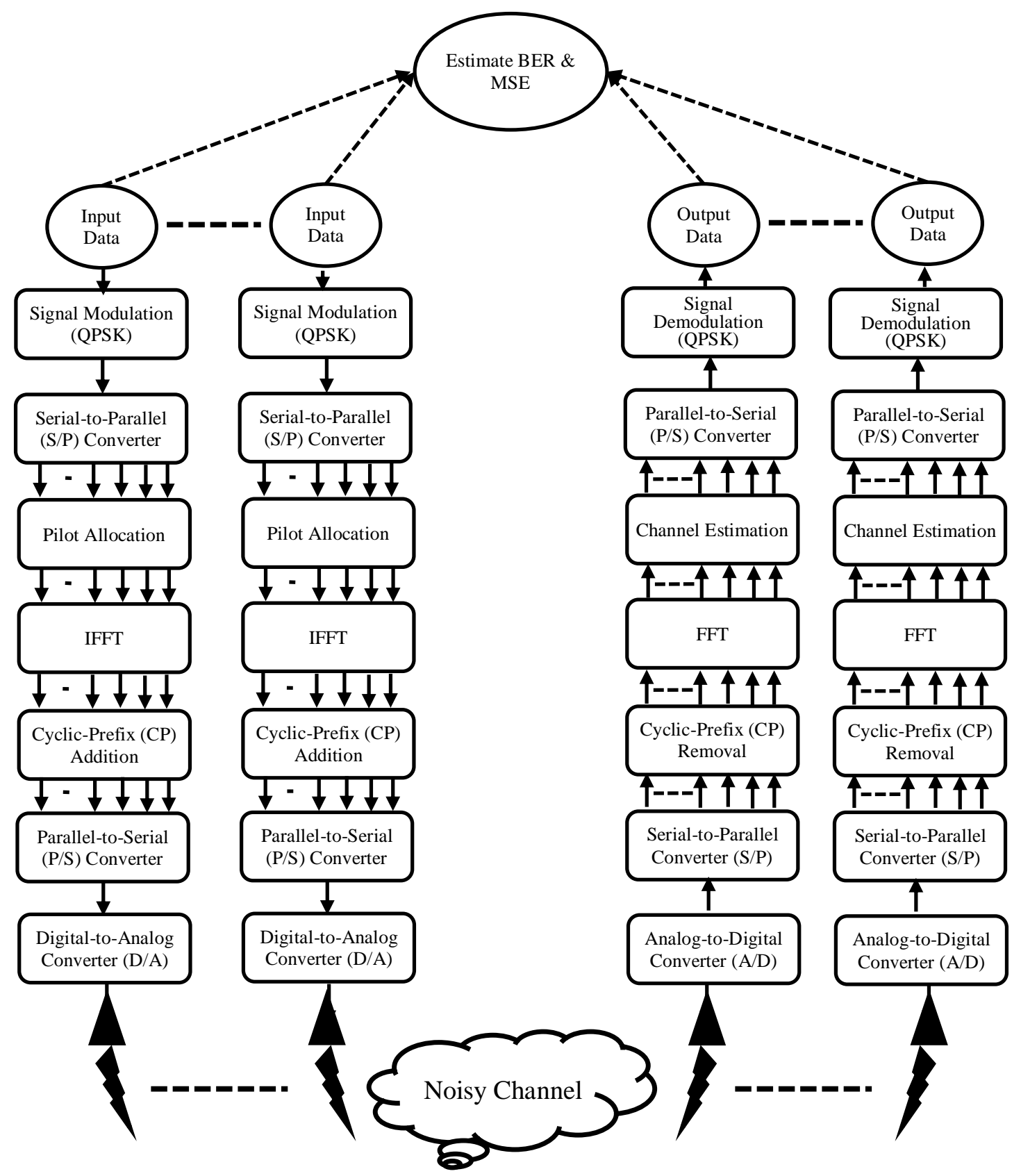

Figure 3. Block diagram of a MIMO-OFDM system.

Figure5 depicts the MSE performance, and once again it can be clearly recognized that the MSE decreases as SNR increasing for all techniques. The comb-type and SIP techniques overwhelm the performance of STBC and SFBC especially at SNR $>5 \mathrm{~dB}$. The comb-type accomplishes slightly better performance than the SIP. Furthermore, SIP-based channel estimation requires redesign of pilot symbols for different transmit antennas and different subcarriers. Hence, the pilot patterns of SIP channel estimation is not flexible compared to that of comb-type channel estimation. The curves of MSE performance in Figure 5 for STBC and SFBC almost agree with the curves of BER performance in Figure4, where SFBC performs better than STBC. 


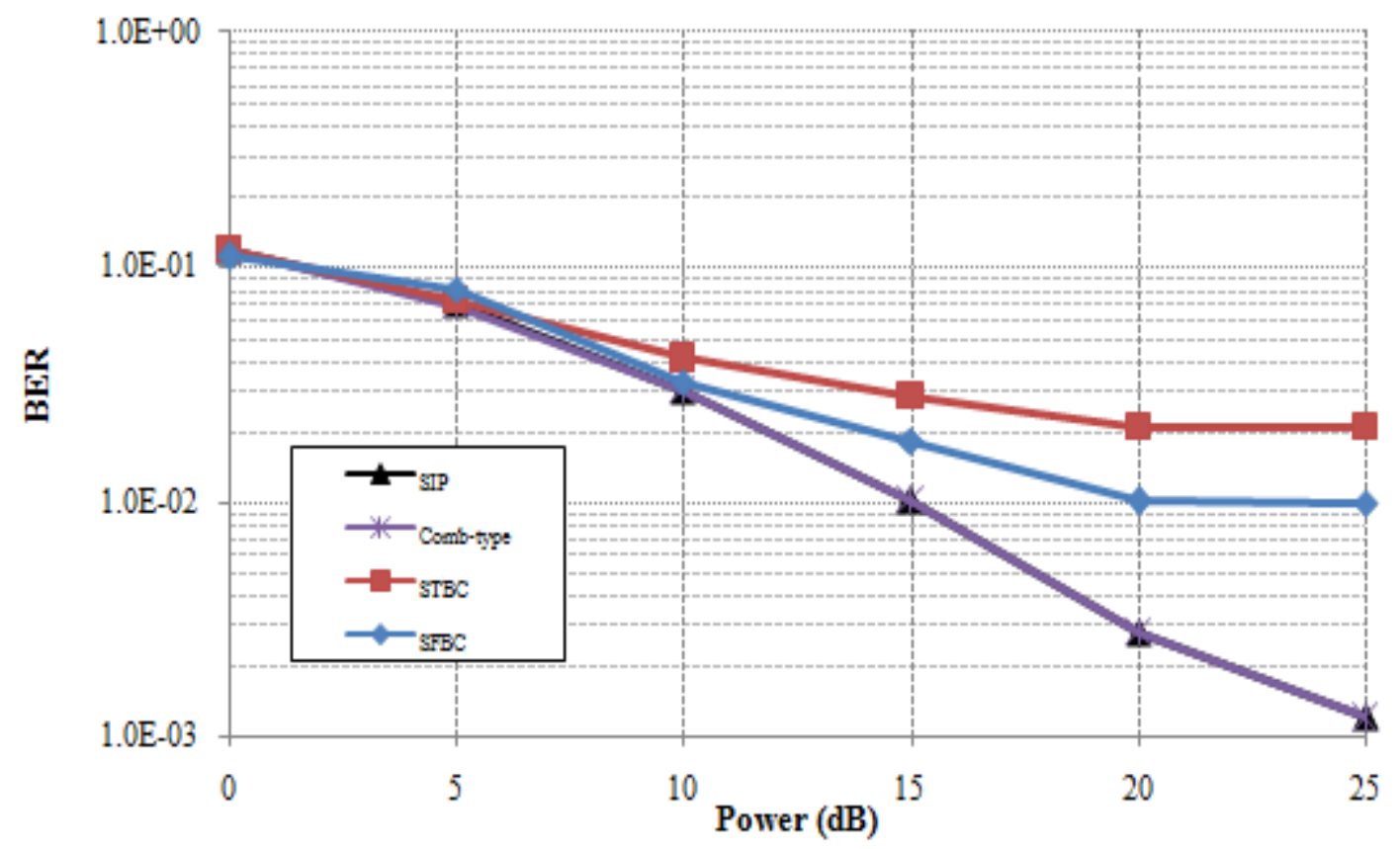

Figure 4. BER vs. SNR $\left(L=8, f_{d} T_{O F D M}=10^{-2}\right)$.

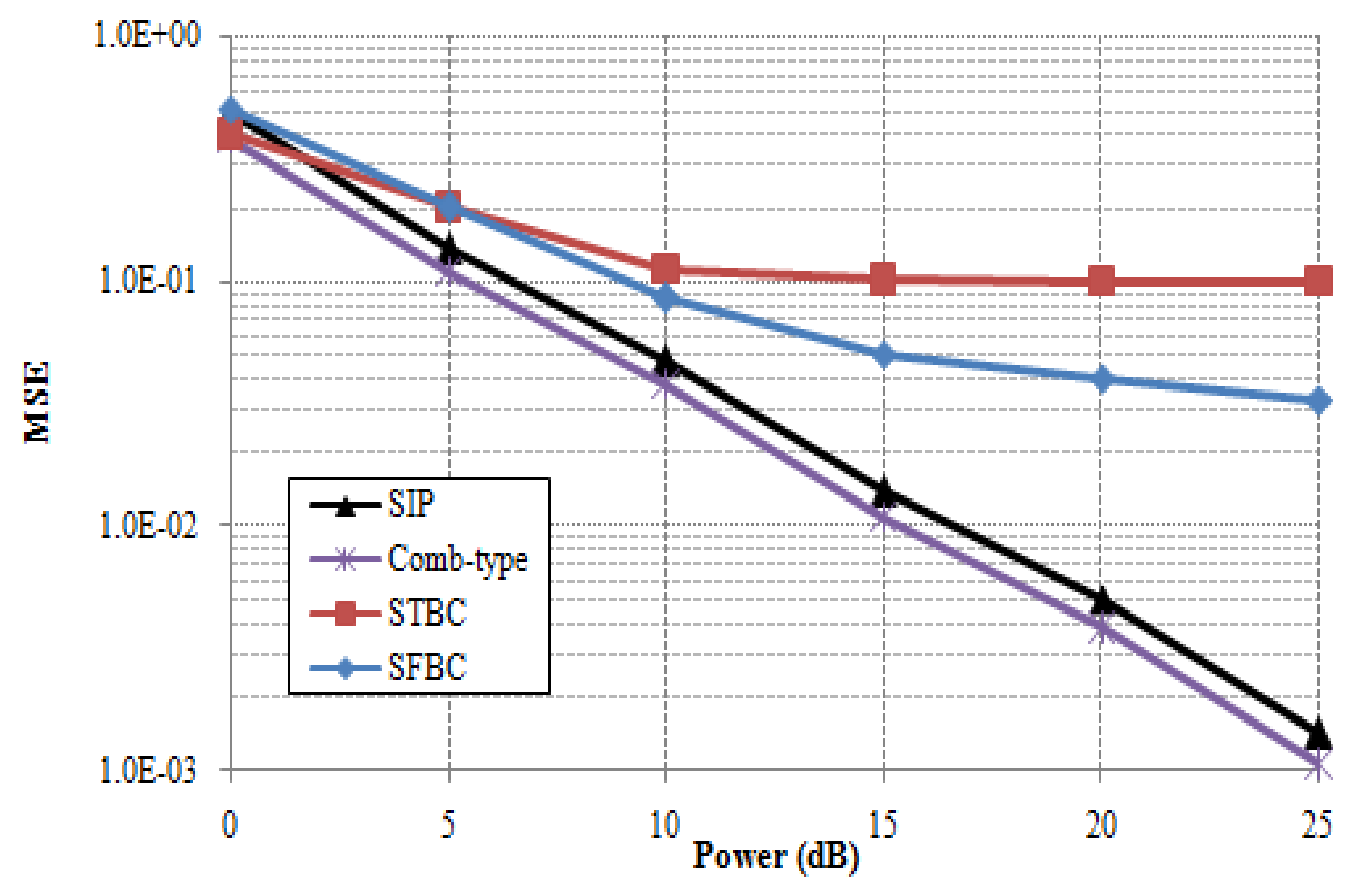

Figure 5. MSE vs. SNR $\left(L=8, f_{d} T_{\text {OFDM }}=10^{-2}\right)$.

\section{Conclusions}

The simulation results demonstrate that comb-type and SIP channel estimation techniques have better performance in terms of BER and MSE (lowest values) as compared to SFBC and STBC techniques for all power range; and SFBC technique provides better performance than STBC. 
Practically, comb-type and SIP outperform both SFBC and STBC significantly, as well as they are more robust and promising. Furthermore, comb-type channels estimation works better than SIP with the same number of pilots, which implies they have the same number of data subcarriers. Based on the simulation results for BER and MSE, the four investigated techniques can be ranked as follows: comb-type, SIP, SFBC, and finally STBC.

The main recommendations for future work are to investigate the effects of the number of subcarrier $\left(N_{s}\right)$, number of pilots $\left(N_{p}\right)$, channel length $(L)$, and normalized Doppler frequency $\left(f_{d} T_{\text {OFDM }}\right)$ on the performance of these techniques. Furthermore, it is also valuable to compare the performance of these techniques against other channel estimation techniques in various wireless communication environments and system conditions.

\section{REFERENCES}

[1] Yong Soo Cho, Jaekwon Kim, Won Young Yang, Chung G. Kang. MIMO-OFDM Wireless Communications with MATLab, John Wiley \& Sons, August 2010.

[2] Sunho Park, Byonghyo Shim, and Jun Won Choi. Iterative Channel Estimation Using Virtual Pilot Signals for MIMO-OFDM Systems. IEEE Transactions on Signal Processing, Vol. 63, Issue: 12, pp. 3032 - 3045, June 2015.

[3] Nisha Achra, Garima Mathur, R. P. Yadav. Performance Analysis of MIMO-OFDM System for Different Modulation Schemes under Various Fading Channels. International Journal of Advanced Research in Computer and Communication Engineering, Vol. 2, Issue 5, May 2013.

[5] V. Sivanagaraju and P. Siddaaih. Comprehensive Analysis of BER and SNR in OFDM Systems. International Journal of Innovative Research in Computer and Communication Engineering (IJIRCCE), Volume 2, Issue 2, February 2014.

[6] Nitin Kumar Chourasiya and Aman Saraf. A Review Channel Estimation Technique for MIMOOFDM Wireless Systems. International Journal on Emerging Technologies, Vol. 8, No. 1, pp. 20-24, 2017.

[7] Vishal Sharma and Harleen Kaur. On BER Evaluation of MIMO-OFDM Incorporated Wireless System. International Journal for Light and Electron Optics, Vol. 127, Issue 1, pp. 203-205, January 2016.

[8] Vijaykumar Katgi. Comparison of MIMO OFDM System with BPSK and QPSK Modulation. International Journal on Emerging Technologies, Vol. 6, No. 2, pp. 188-192, 2015.

[9] Sharief Nasr Abdel-Razeq, Areej Salah Al-Azzeh, Rawan Yousef Ayyoub. Study of QPSK Modulator and Demodulator in Wireless Communication System Using MATLab. International Journal of Interactive Mobile Technologies (iJIM), Vol. 7, No. 2, pp. 4-8, 2013. http://dx.doi.org/10.3991/ijim.v7i2.2239.

[10] Mathuranathan Viswanathan. Digital Modulations using MATLab: Build Simulation Models from Scratch. E-book, June, 2017.

[11] Sagar Somashekar, Naveen K.D. Venkategowda, and Aditya K.Jagannatham. Bandwidth Efficient Optimal Superimposed Pilot Design for Channel Estimation in OSTBC-Based MIMO-OFDM Systems. Journal of Physical Communication, Vol. 26, pp. 185-195, February 2018.

[12] Umesha G B and M N Shanmukha Swamy. Comb-Type Pilot Arrangement Based Channel Estimation for Spatial Multiplexing MIMO-OFDM Systems. International Research Journal of Engineering and Technology (IRJET), Vol. 5, Issue 2, pp. 641-645, February 2018. 
[13] Gaurav Maurya and Pramod Patel. An Extensive Review on STBC for MIMO-OFDM System. International Journal of Emerging Technology and Advanced Engineering (IJETAE), Vol. 4, Issue-8, pp. 352-357, August 2014.

[14] Neeraj Shrivastava and Aditya Trivedi. Combined Beamforming with Space-Time-Frequency Coding for MIMO-OFDM Systems. AEU - International Journal of Electronics and Communications, Vol. 69, Issue 6, pp. 878-883, June 2015.

[15] Madhvi Jangalwa. Performance Analysis of MIMO-OFDM system with Zero Forcing Receiver. International Journal of Multidisciplinary and Current Research, September-October Issue, 2013.

[16] Mamunur Rashid and Saddam Hossain. A Potent MIMO-OFDM System Designed for Optimum BER and its Performance Analysis in AWGN Channel. International Journal of Engineering Science Invention (IJESI), Vol. 4, Issue 8, pp. 44-50, August 2015.

[17] Atul Kumar Pandey, Santosh Sharma, and Neetu Sikarwar. Improvement of BER with The Help of MIMO-OFDM using STBC Code Structure. International Journal of Engineering Research \& Technology (IJERT), Vol. 2, Issue 5, May 2013.

[18] Niharika Sethy and Subhakanta Swain. BER Analysis of MIMO-OFDM System in Different Fading Channe. International Journal of Application or Innovation in Engineering and Management, Vol. 2, Issue 4, pp. 405-409, April 2013.

[19] B. Siva Kumar Reddy and B. Lakshmi. BER Analysis with Adaptive Modulation Coding in MIMOOFDM for WiMAX using GNU Radio. International Journal Wireless and Microwave Technologies, November 2014.

[20] Srishtansh Pathak and Himanshu Sharma. Channel Estimation in OFDM Systems. International Journal of Advanced Research in Computer Science and Software Engineering (IJARCSSE), Vol.3, No.3, pp. 312-327, 2013.

[21] S. Patil and A. N. Jadhav. Channel Estimation Using LS and MMSE Estimators. KIET International Journal of Communications \& Electronics, Vol. 2, No.1, pp. 51-55, April 2014.

[22] Himanshi Jain and Vikas Nandal. A Comparison of Various Channel Estimation Techniques to Improve Fading Effects in MIMO over Different Fading Channels. International Journal of Current Engineering and Technology (IJCET), Vol. 6, No. 4, pp. 1382-1386, 2016.

[23] Omar Daoud, Qadri Hamarsheh, and Wael Al-Sawalmeh. MIMO-OFDM Systems Performance Enhancement Based Peaks Detection Algorithm. International Journal of Interactive Mobile Technologies (iJIM), Vol. 7, No. 3, pp. 4-8, 2013. http://dx.doi.org/10.3991/ijim.v7i3.2302.

[24] Omar Daoud and Omar Alani. Robotic Mobile System's Performance-Based MIMO-OFDM Technology. International Journal of Interactive Mobile Technologies (iJIM), Vol. 3, pp.12-17, 2009. http://online-journals.org/index.php/i-jim/article/view/923.

[25] Nimay Ch. Giri, Anwesha Sahoo, J. R. Swain, P. Kumar, A. Nayak, P. Debogoswami. Capacity and Performance Comparison of SISO and MIMO System for Next Generation Network (NGN). International Journal of Advanced Research in Computer Engineering \& Technology (IJARCET), Vol. 3, Issue 9, pp. 30131-3035, 2014.

[26] O. Longoria-Gandara, R. Parra-Michel, R.Carrasco-Alvarez, and E. Romero-Aguirre. Iterative MIMO Detection and Channel Estimation Using Joint Superimposed and Pilot-Aided Training. Journal of Mobile Information Systems, Vol. 2016, Article ID 3723862, 2016. 


\section{AuTHORS}

Woud M. Abed is a member of academic staff at the Department of Basic Sciences, College of Dentistry, University of Baghdad (Baghdad, Iraq). She received her B.Sc degree in Computer Science from the Department of Computer Science, Alrafidain University College (Baghdad, Iraq) in 2003, and her M.Sc degree in Computer Networks, Informatics Institute for Higher Studies, University of Technology (Baghdad, Iraq) in 2005. Her research interests include: robotic, genetic algorithms, cryptography and steganography, image processing, and

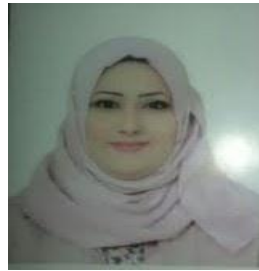
computer security.

Raghad K. Mohammed is serving as a member of academic staff at the Department of Basic Sciences, College of Dentistry, University of Baghdad (Baghdad, Iraq). She received her B.Sc degree in Computer Science from the Department of Computer science, Alrafidain University College (Baghdad, Iraq) in 2003, and her M.Sc degree in Computer Networks, Informatics Institute for Higher Studies, University of Technology (Baghdad, Iraq) in 2005. Her research interests include cryptography and steganography, image processing, and information and network security.

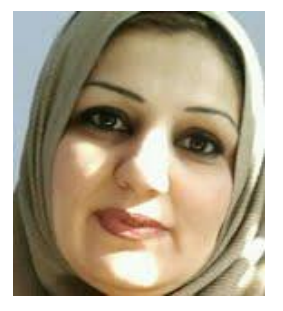

